\title{
Species and inter-individual differences in metabolic capacity of di(2-ethylhexyl)phthalate (DEHP) between human and mouse livers
}

\author{
Yuki Ito $\cdot$ Michihiro Kamijima $\cdot$ Chie Hasegawa $\cdot$ \\ Masahiro Tagawa $\cdot$ Toshio Kawai $\cdot$ Mio Miyake \\ Yumi Hayashi · Hisao Naito · Tamie Nakajima
}

Received: 28 June 2013/Accepted: 4 September 2013/Published online: 28 September 2013

(C) The Japanese Society for Hygiene 2013

\begin{abstract}
Objectives This study was conducted to assess interspecies and inter-individual differences in the metabolism of di(2-ethylhexyl)phthalate (DEHP) in humans and mice. Methods The activities of four DEHP-metabolizing enzymes [lipase, UDP-glucuronocyltransferase (UGT), alcohol dehydrogenase (ADH), aldehyde dehydrogenase (ALDH)] were measured in the livers of 38 human subjects of various ages and in eight 129/Sv male mice.

Results Microsomal lipase activity was significantly lower in humans than in mice. The $V_{\max } / K_{\mathrm{m}}$ value in humans was one-seventh of that in mice, microsomal UGT activity in humans was a sixth of that in mice, and cytosolic ALDH activity for 2-ethylhexanal in humans was one-half of that in mice. In contrast, ADH activity for 2-ethylhexanol was twofold higher in humans than in mice. The total amount of DEHP urinary metabolites and the concentration of mono(2ethylhexyl)phthalate (MEHP) were much higher in intact mice than in the U.S. general population based on data reported elsewhere, regardless of the similar estimated DEHP intake between these mice and the human reference population. However, mono(2-ethyl-5-oxo-hexyl)phthalate (5oxoMEHP) and mono(2-ethyl-5-carboxypentyl)phthalate (5cx-
\end{abstract}

Y. Ito $(\bowtie) \cdot$ M. Kamijima $\cdot$ C. Hasegawa · M. Miyake Department of Occupational and Environmental Health,

Nagoya City University Graduate School of Medical Sciences,

Nagoya 467-8601, Japan

e-mail: yukey@med.nagoya-cu.ac.jp

M. Tagawa · Y. Hayashi · H. Naito · T. Nakajima

Department of Occupational and Environmental Health,

Nagoya University Graduate School of Medicine, Nagoya, Japan

T. Kawai

Osaka Occupational Health Service Center, Japan Industrial

Safety and Health Association, Kawachinagano, Japan
MEPP) levels were higher in the latter than in the former. Of note, inter-subject variability in the activities of all enzymes measured was 10-26-fold.

Conclusion The inter-individual variation in the metabolism of DEHP in humans may be greater than the difference between mice and humans (inter-species variation), and both may affects the risk assessment of DEHP.

Keywords Di(2-ethylhexyl)phthalate · Human · Metabolism · Liver · Enzyme activities

$\begin{array}{ll}\text { Abbreviations } \\ \text { ADH } & \text { Alcohol dehydrogenase } \\ \text { ALDH } & \text { Aldehyde dehydrogenase } \\ \text { BMI } & \text { Body mass index } \\ \text { CYPs } & \text { Cytochrome P450s } \\ \text { 5cx-MEPP } & \text { Mono(2-ethyl-5-carboxypentyl)phthalate } \\ \text { 2cx-MMHP } & \text { Mono(2-carboxymethylhexyl)phthalate } \\ \text { DEHP } & \text { Di(2-ethylhexyl)phthalate } \\ \text { 2-EH } & \text { 2-Ethylhexanol } \\ \text { 2-EHA } & \text { 2-Ethylhexanoic acid } \\ \text { HAB } & \text { Human and Animal Bridging } \\ \text { K } & \text { Michaelis constant } \\ \text { MEHP } & \text { Mono(2-ethylhexyl)phthalate } \\ \text { 5OH-MEHP } & \text { Mono(2-ethyl-5-hydroxyhexyl)phthalate } \\ 50 x 0-M E H P & \text { Mono(2-ethyl-5-oxo-hexyl)phthalate } \\ \text { UGT } & \text { UDP-glucuronocyltransferase } \\ V_{\text {max }} & \text { Maximal velocity }\end{array}$

\section{Introduction}

Di(2-ethylhexyl)phthalate (DEHP) is widely used as a plasticizer to improve the plasticity and elasticity of 
polyvinyl chloride (PVC) products. Generally, these final products contain between 10 and $60 \% \operatorname{DEHP}(w / w)$. DEHP is widespread in the environment because of its nonchemical binding to polymer products, which results in the general population being exposed to this chemical in on a daily basis. In fact, DEHP metabolites were detected in all urine samples obtained from subjects participating in the U.S. National Health and Nutrition Examination Survey [1].

Humans are mainly exposed to DEHP via ingestion of food. Once absorbed into the body, DEHP is rapidly hydrolyzed to mono(2-ethylhexyl)phthalate (MEHP) and 2-ethylhexanol (2-EH) by the catalytic action of lipase [2, 3]. A part of MEHP is further oxidized by various cytochrome P450s (CYPs), followed by alcohol dehydrogenase $(\mathrm{ADH})$ or aldehyde dehydrogenase $(\mathrm{ALDH})$, to produce dicarboxylic acid or ketones. The remaining MEHP is excreted in the urine directly or in the conjugation form by the catalytic action of UDP-glucuronocyltransferase (UGT). 2-EH is metabolized mainly to carboxylic acid [mainly 2-ethylhexanoic acid, 2-EHA] via 2-ethylhexanal by the catalytic action of ADH and ALDH [2]. The 2-EHA formed is further oxidized to a dicarboxylic acid in a way similar to MEHP oxidation.

In earlier studies we clarified species differences in enzyme activities involved in DEHP metabolism among mice, rats, and marmosets as a reference to primates $[4,5]$. Of these enzymes, the most prominent difference among the studied species was that in lipase activity, and we noted that lipase activity significantly influenced the internal amount of MEHP [4, 5]. The mean lipase activity in the liver was found to be considerably lower in marmosets than in mice [5]. The maximum velocity value $\left(V_{\max }\right)$ of the reaction was lowest in marmosets, but the Michaelis constant $\left(K_{\mathrm{m}}\right)$ value in this species was the highest. Therefore, the $V_{\max } / K_{\mathrm{m}}$ ratio in marmosets was $1 / 241$-fold that of mice. However, it is questionable whether lipase activity in marmosets can be considered to be the same as that in humans. Indeed, the National Toxicology Program and Health Assessment and Translation (Formerly Center for the Evaluation of Risks to Human Reproduction) expert panel stated in their review that there is a critical lack of data on human metabolism across ages; the exact differences in the metabolic capacity between humans and animal models remain to be elucidated [6] with the exception of CYPs [7]. Moreover, inter-individual differences among humans have not been examined.

In the study reported here, we investigated the activities of four enzymes (lipase, UGT, ADH, ALDH) involved in the metabolism of DEHP in human liver and compared the activities with those of mice. Our results confirm large inter-individual differences in the respective metabolic enzyme activities, as well as obvious species differences in metabolic capacities and pathways.

\section{Materials and methods}

\section{Subjects}

This study's protocol was approved by the Ethics Committees of Nagoya City University Graduate School of Medical Sciences and the nonprofit organization Human and Animal Bridging (HAB) Research Organization (Chiba, Japan). Frozen human livers that had been removed from brain-dead donors were used in this study. These livers did not match recipients for transplant in the USA and were procured by the U.S. National Human Tissue Resource Center. The livers were removed between 1996 and 2010 from the donors and obtained from the HAB Research Organization. In each case, informed consent for research use was obtained from the closest family member in written form in each hospital. To compare age-, race-, and sex-related effects, we chose the widest range of subjects possible.

The animal study was conducted according to the Guidelines for Animal Experiments of The Nagoya University Animal Center. Animals were housed in a cage in a clean room under controlled temperature and relative humidity conditions, with a 12/12-h light/dark light regime. $129 / \mathrm{Sv}$ mice were mated in the animal center, and 20- to 21 -week-old male offspring were used $(n=8)$. All mice were sacrificed by exsanguination from the abdominal aorta under pentobarbital anesthesia, following which the livers were removed and stored at $-80{ }^{\circ} \mathrm{C}$ until use.

All available data for the 38 human subjects are shown in Table 1. Most subjects were male non-Hispanic whites between 16 and 80 years of age. Five female non-Hispanic whites were all in their forties. In addition to the livers from non-Hispanics, livers from five Hispanics, three African Americans, and one Asian were available. Most subjects died from cerebrovascular disease. The mean body mass index (BMI) was $29.6 \pm 8.8$. We obtained information from the HAB Research Organization on the drinking and smoking habits and drug use of the donors during the 1 year preceding their death; this information was gathered through interviews with the family members. The frequency of drinking was categorized into four groups: always, frequently, occasionally, and seldom. However, accurate data on alcohol consumption in most subjects were not available.

\section{Sample preparation}

Liver homogenates and microsomal and cytosolic fractions were prepared according to the method of Wang et al. [8]. The protein concentrations were measured using a Pierce ${ }^{\circledR}$ BCA Protein Assay Kit (Thermo Scientific, Rockford, IL). 
Table 1 Characteristics of all 38 liver donors

\begin{tabular}{|c|c|c|c|c|c|c|c|c|}
\hline Race & Sex & $\begin{array}{l}\text { Age } \\
\text { (years) }\end{array}$ & Causes of death & BMI & Drinking status & $\begin{array}{l}\text { Drinking } \\
\text { frequency }\end{array}$ & $\begin{array}{l}\text { Smoking } \\
\text { status }\end{array}$ & $\begin{array}{l}\text { Recent drug } \\
\text { use }\end{array}$ \\
\hline Non-Hispanic white & Male & 16 & Accident & 39.4 & Non-Drinker & - & Non-smoker & No \\
\hline Non-Hispanic white & Male & 25 & Accident & 22.1 & Drinker & Frequently & Smoker & Yes \\
\hline Non-Hispanic white & Male & 35 & Accident & 27.1 & Drinker & Frequently & Smoker & Yes \\
\hline Non-Hispanic white & Male & 36 & Accident & 28.7 & Drinker & Occasionally & Smoker & Yes \\
\hline Non-Hispanic white & Male & 36 & Cerebrovascular disease & 41.8 & Drinker & Occasionally & Ex-smoker & No \\
\hline Non-Hispanic white & Male & 38 & Cerebrovascular disease & 34.4 & Drinker & Frequently & Smoker & No \\
\hline Non-Hispanic white & Male & 38 & Accident & 19.4 & Drinker & Occasionally & Smoker & No \\
\hline Non-Hispanic white & Male & 41 & Accident & 24.2 & Non-drinker & - & Non-smoker & No \\
\hline Non-Hispanic white & Male & 44 & Cerebrovascular disease & 21.8 & Non-drinker & - & Smoker & No \\
\hline Non-Hispanic white & Male & 46 & Accident & 24.2 & Drinker & Frequently & Smoker & Yes \\
\hline Non-Hispanic white & Male & 46 & Accident & 28.4 & Drinker & Seldom & Smoker & No \\
\hline Non-Hispanic white & Male & 47 & Aneurysm & 32.1 & Drinker & Frequently & Smoker & No \\
\hline Non-Hispanic white & Male & 51 & Cerebrovascular disease & 20.2 & Drinker & Occasionally & Smoker & No \\
\hline Non-Hispanic white & Male & 52 & Cerebrovascular disease & 40.4 & Non-Drinker & - & Ex-smoker & No \\
\hline Non-Hispanic white & Male & 53 & Cerebrovascular disease & 27.3 & Drinker & Frequently & Ex-smoker & No \\
\hline Non-Hispanic white & Male & 56 & Subdural hemorrhage & 28.4 & Drinker, abuse & Always & Non-smoker & No \\
\hline Non-Hispanic white & Male & 56 & Cerebrovascular disease & 29.9 & Drinker & Frequently & Smoker & No \\
\hline Non-Hispanic white & Male & 60 & Cerebrovascular disease & 62.1 & Drinker & N.I. & Smoker & No \\
\hline Non-Hispanic white & Male & 63 & Cerebrovascular disease & 28.4 & Drinker & Frequently & Smoker & No \\
\hline Non-Hispanic white & Male & 65 & Cerebrovascular disease & 25.5 & Drinker & Frequently & Non-smoker & No \\
\hline Non-Hispanic white & Male & 68 & Cerebrovascular disease & 36.1 & Non-Drinker & - & Ex-smoker & No \\
\hline Non-Hispanic white & Male & 71 & Cerebrovascular disease & 23.7 & Non-Drinker & - & Ex-smoker & Yes \\
\hline Non-Hispanic white & Male & 71 & Cerebrovascular disease & 26.2 & Non-Drinker & - & Non-smoker & No \\
\hline Non-Hispanic white & Male & 80 & Cerebrovascular disease & 24.7 & Non-Drinker & - & Non-smoker & No \\
\hline Non-Hispanic white & Female & 41 & Cerebrovascular disease & 19.9 & Drinker & Occasionally & Smoker & No \\
\hline Non-Hispanic white & Female & 41 & Cerebrovascular disease & 27.7 & Drinker & Seldom & Smoker & Yes \\
\hline Non-Hispanic white & Female & 45 & Cerebrovascular disease & 41.5 & Non-drinker & - & Non-smoker & No \\
\hline Non-Hispanic white & Female & 47 & Cerebrovascular disease & 24.5 & Drinker & Occasionally & Smoker & No \\
\hline Non-Hispanic white & Female & 49 & Subdural hemorrhage & 34.8 & Non-drinker & - & Non-smoker & No \\
\hline Asian & Male & 57 & Cerebrovascular disease & 45.0 & Non-drinker & - & Non-smoker & No \\
\hline African-American & Male & 62 & Cerebrovascular disease & 31.4 & Drinker & N.I. & Non-smoker & No \\
\hline African-American & Female & 34 & Cerebrovascular disease & 37.9 & Drinker & Seldom & Smoker & Yes \\
\hline African-American & Female & 52 & Cerebrovascular disease & 22.8 & Non-drinker & - & Non-smoker & No \\
\hline Hispanic & Male & 24 & Ischemic heart disease & 16.9 & Non-drinker & - & Non-smoker & No \\
\hline Hispanic & Male & 49 & Accident & 29.3 & Drinker & Frequently & Ex-smoker & No \\
\hline Hispanic & Male & 55 & Cerebrovascular disease & 23.8 & Drinker, abuse & Always & Ex-smoker & No \\
\hline Hispanic & Male & 60 & Cerebrovascular disease & 22.1 & Drinker & Occasionally & Smoker & No \\
\hline Hispanic & Female & 35 & Cerebrovascular disease & 31.6 & Drinker & Seldom & Non-smoker & No \\
\hline
\end{tabular}

$B M I$ Body mass index, N.I. no information

\section{Measurement of enzyme activities}

Lipase activity in the liver microsomes was determined by measuring the rate of MEHP formation from DEHP, as described elsewhere [5, 9]. As an internal standard, $0.5 \mathrm{nmol}$ deuterated-MEHP was used. Lipase kinetics in the range of DEHP concentration $(20-5000 \mu \mathrm{M}$ for humans and $10-1000 \mu \mathrm{M}$ for mice) were determined according to a previously published method [5]. Briefly, pooled hepatic microsomes were used for measuring lipase activity, and $V_{\max }$ and $K_{\mathrm{m}}$ values for DEHP were determined from Lineweaver-Burk plots.

UGT activity was measured using the UGT-Glo ${ }^{\mathrm{TM}}$ Assay kit (Promega, Madison, WI) according to the 
manufacturer's protocol. We used a final concentration of $0.1 \mathrm{mg} / \mathrm{ml}$ hepatic microsomes and $50 \mu \mathrm{M}$ of multienzyme substrate and incubated this assay mixture at $37^{\circ} \mathrm{C}$ for $15 \mathrm{~min}$. The multienzyme substrate has a high affinity for many UGT enzymes, especially UGT 1A1, 1A8, 1A9, $1 \mathrm{~A} 10,2 \mathrm{~B} 7$, and 2B15. MEHP or MEHP oxidative metabolites were glucuronized by those UGT isoenzymes and, therefore, the results from the UGT assay were considered to be applicable to the case of MEHP.

$\mathrm{ADH}$ and $\mathrm{ALDH}$ activities were measured using the cytosol fraction $(100 \mu \mathrm{g})$ and homogenates $(200 \mu \mathrm{g})$, respectively. These activities were measured according to the method of Wang et al. [8] with slight modifications in substrate concentration. As substrates for ADH and ALDH, $10 \mu \mathrm{M}$ of 2 -EH and $80 \mu \mathrm{M}$ of 2-ethylhexanal, respectively, were used.

\section{Measurement of urinary metabolites}

In order to investigate total DEHP metabolites and each element, urine excreted for $24 \mathrm{~h}$ from 129/Sv male mice (age 20-21 weeks) was collected and stored at $-80{ }^{\circ} \mathrm{C}$ until use. An aliquot $(500 \mu \mathrm{l})$ of urine was mixed with $1110 \mu \mathrm{l}$ of reaction mixture containing a final concentration of $45 \mathrm{mM}$ acetate buffer ( $\mathrm{pH} 4.8$ ), $60 \mathrm{U}$ of $\beta$-glucuronidase [10], and $100 \mathrm{ng}$ deuterated-MEHP as an internal standard and incubated at $37{ }^{\circ} \mathrm{C}$ overnight, following which the urinary metabolites of DEHP were extracted with ethyl acetate and converted to tert-butyldimethylsilyl derivatives by adding $N$-methyl- $N$-(tert-butyldimethylsilyl) trifluoroacetamide, as reported previously [5]. Analytical methods were also conducted according to Ito et al. [5] with slight modifications. Because the concentration of mono(2-ethyl-5-carboxypentyl)phthalate (5cx-MEPP) was under the detection limit $(5 \mathrm{ng} / \mathrm{ml})$, we used the value of half the limit [11].
Statistical analysis

Statistical analysis was carried out using the JMP 5.0.1J software package (SAS Institute, Cary, NC). Values of $P<0.05$ were considered to indicate statistical significance. Comparisons were conducted using the analysis of variance and the Tukey-Kramer HSD post hoc test or Student's $t$ test. The correlations among enzyme activities were analyzed using Spearman's rank methods, and their rank correlation coefficients $\left(r_{\mathrm{s}}\right)$ were shown when they were statistically significant.

\section{Results}

Lipase activity and MEHP concentration in the liver

Lipase activity in the livers is shown in Table 2. Lipase activity in our human subjects was clearly lower than that in mice regardless of sex, age, or race differences. In addition, alcohol intake or its frequency, smoking habit, drug use, BMI, cause of death, and the time elapsed before the livers were frozen also did not influence lipase activity (data not shown). The mean and $95 \%$ confidence interval (CI) of the activities were 1.21 (95\% CI 1.03-1.40) nmol/ $\mathrm{mg} / \mathrm{min}$ in humans and 6.22 (95\% CI 5.31-7.12) $\mathrm{nmol} / \mathrm{mg} /$ min in mice. The inter-individual difference was 9.8 -fold (maximum $3.51 \mathrm{nmol} / \mathrm{mg} / \mathrm{min}$, minimum $0.36 \mathrm{nmol} / \mathrm{mg} /$ min). Even the lowest observed activity in the mouse liver was higher than the highest observed activity in the human liver.

Species differences in lipase $V_{\max }$ and $K_{\mathrm{m}}$ for DEHP calculated from Lineweaver-Burk plots (Fig. 1) are shown in Table 3. The $V_{\max }$ of lipase activity for DEHP was 3.6-fold higher in mice than in humans. In contrast,

Table 2 Lipase, UGT, ADH and ALDH activities (nmol/mg protein/min) according to species, race, age-group and sex

\begin{tabular}{|c|c|c|c|c|c|c|c|c|c|c|c|c|c|c|c|c|}
\hline \multirow{2}{*}{ Species } & \multirow{2}{*}{ Race/Strain } & \multirow{2}{*}{ Age-group } & \multirow{2}{*}{ Sex } & \multirow{2}{*}{$\mathrm{N}$} & \multicolumn{3}{|c|}{ Lipase } & \multicolumn{3}{|c|}{ UGT } & \multicolumn{3}{|c|}{$\mathrm{ADH}$} & \multicolumn{3}{|c|}{ ALDH } \\
\hline & & & & & Mean & Min - Max & $(95 \% \mathrm{CI})$ & Mean & Min - Max & $(95 \% \mathrm{CI})$ & Mean & Min - Max & $(95 \% \mathrm{Cl})$ & Mean & Min - Max & $(95 \% \mathrm{CI})$ \\
\hline Human & & $16-80$ & $\mathrm{M}, \mathrm{F}$ & 38 & 1.21 & $0.36-3.51$ & $(1.03-1.40)$ & 22.2 & $3.38-58.94$ & $(17.40-27.07)$ & $8.6-$ & $1.5-15.5$ & $(7.4-9.8)$ & 3.6 & $0.4-10.5$ & $\overline{(3.0-4.3)}$ \\
\hline & Non-Hispanic white & 16 & $\mathrm{M}$ & 1 & 1.73 & - & - & 5.5 & - & - & 8.4 & - & - & 10.5 & - & - \\
\hline & Non-Hispanic white & 25 & M & 1 & 0.95 & - & - & 8.8 & - & - & 4.7 & - & - & 4.0 & - & - \\
\hline & Non-Hispanic white & 30 's & M & 5 & 1.08 & $0.36-1.62$ & $(0.48-1.69)$ & 25.1 & $9.95-39.90$ & $(7.57-42.69)$ & 9.8 & $6.3-12.8$ & $(6.9-12.7)$ & 3.6 & $2.0-6.2$ & $(1.7-5.6)$ \\
\hline & Non-Hispanic white & 40 's & M & 5 & 0.96 & $0.80-1.15$ & $(0.79-1.13)$ & 22.3 & $11.44-33.48$ & $(12.22-32.33)$ & 8.2 & $4.2-12.0$ & $(3.9-12.6)$ & 3.2 & $1.4-5.0$ & $(1.6-4.9)$ \\
\hline & Non-Hispanic white & 40 's & $\mathrm{F}$ & 5 & 1.30 & $0.53-2.05$ & $(0.54-2.05)$ & 22.8 & $3.38-58.94$ & $(-4.80-50.32)$ & 8.2 & $6.3-10.6$ & $(5.4-10.9)$ & $5.07 *$ & $4.0-5.8$ & $(4.2-5.8)$ \\
\hline & Non-Hispanic white & 50 's & M & 5 & 1.17 & $0.65-1.74$ & $(0.63-1.72)$ & 24.3 & $9.04-54.62$ & $(2.22-46.34)$ & 7.1 & $3.0-10.6$ & $(3.0-11.2)$ & $3.2\rfloor *$ & $1.4-5.8$ & $(1.0-5.3)$ \\
\hline & Non-Hispanic white & 60 's & M & 4 & 1.20 & $0.87-1.62$ & $(0.70-1.69)$ & 15.1 & $6.76-23.32$ & $(4.27-25.98)$ & 9.7 & $1.5-13.2$ & $(1.0-18.5)$ & 3.4 & $2.8-4.6$ & $(2.1-4.7)$ \\
\hline & Non-Hispanic white & $71-80$ & M & 3 & 2.08 & $0.55-3.51$ & $(-1.60-5.76)$ & 37.0 & $5.68-56.02$ & $(-30.91-104.93)$ & 14.5 & $13.1-15.5$ & $(11.4-17.6)$ & 4.6 & $1.0-7.2$ & $(-3.4-12.5)$ \\
\hline & Asian & 57 & M & 1 & 1.01 & - & - & 32.6 & - & - & 3.7 & - & - & 3.6 & - & - \\
\hline & African-American & $34-62$ & $\mathrm{M}, \mathrm{F}$ & 3 & 1.05 & $0.80-1.46$ & $(0.17-1.94)$ & 21.2 & $13.94-33.77$ & $(-5.85-48.32)$ & 9.1 & $7.0-12.1$ & $(2.5-15.7)$ & 2.7 & $1.7-3.2$ & $(0.6-4.8)$ \\
\hline & Hispanic & $24-60$ & $\mathrm{M}, \mathrm{F}$ & 5 & 1.14 & $0.61-1.60$ & $(0.58-1.70)$ & 18.1 & $7.94-30.30$ & $(8.12-28.13)$ & 6.8 & $1.6-11.4$ & $(1.8-11.7)$ & 1.8 & $0.4-3.7$ & $(0.2-3.4)$ \\
\hline Mouse & $129 / \mathrm{Sv}$ & & M & 8 & 6.22 * & $3.91-7.54$ & $(5.31-7.12)$ & 121.9 * & $101.15-128.27$ & $(113.6-130.3)$ & $4.2 \stackrel{*}{-}$ & $3.3-5.0$ & $(3.7-4.7)$ & $5.6 *$ & $4.2-7.4$ & $(4.6-6.6)$ \\
\hline
\end{tabular}

These results were determined by duplicate analyses

$95 \%$ CI $95 \%$ confidence interval, Min minimum, Max maximum

* $P<0.05$ by Tukey-Kramer HSD post hoc test or Student's $t$ test after one-way analysis of variance 


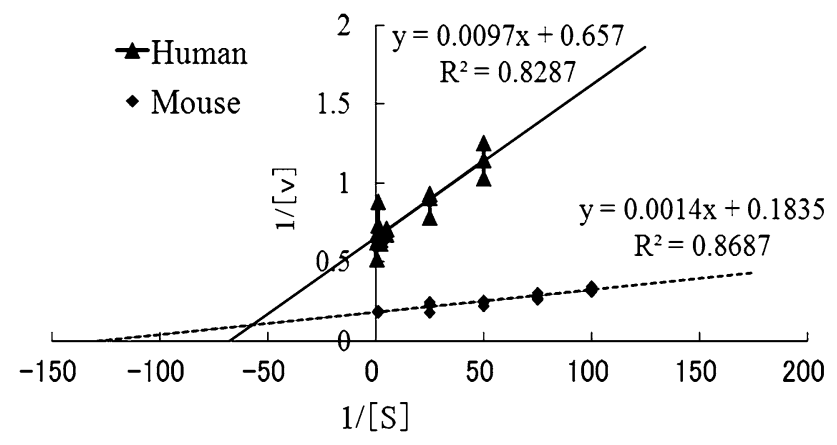

Fig. 1 Lineweaver-Burk plot of lipase activity in hepatic microsomes from human and mouse. Each point represents results from triplicate determinations using pooled microsomes

Table 3 Species difference in the maximum velocity value and Michaelis constant of lipase activity for di(2-ethylhexyl)phthalate in hepatic microsomes from human and mouse

\begin{tabular}{lcc}
\hline Reaction parameters & Human & Mouse \\
\hline$K_{\mathrm{m}}(\mathrm{mmol} / \mathrm{L})$ & 0.0144 & 0.0076 \\
$V_{\max }(\mathrm{nmol} / \mathrm{mg}$ protein $/ \mathrm{min})$ & 1.52 & 5.45 \\
$V_{\max } / K_{\mathrm{m}}$ ratio & 106 & 714 \\
\hline
\end{tabular}

$K_{\mathrm{m}}$ Michaelis constant, $V_{\max }$ maximum velocity value of the reaction Values are presented as the means of the results using three pooled samples per each concentration

the $K_{\mathrm{m}}$ for DEHP was 1.9 -fold lower in mice than in humans. Consequently, 6.7-fold differences were observed in the $V_{\max } / K_{\mathrm{m}}$ ratio (often referred to as an index of intrinsic clearance) between mice and humans. These findings suggest that differences in lipase activity among these species resulted from the different affinity DEHP has for lipase, as well as from the different levels of enzyme expression.

\section{UGT activity}

UGT for multisubstrates are shown in Table 2. Interindividual differences in human UGT actitivity were extremely large, with the highest activity being $58.94 \mathrm{nmol} / \mathrm{mg} / \mathrm{min}$ and the lowest activity being $3.38 \mathrm{nmol} / \mathrm{mg} / \mathrm{min}$. UGT activity in mice $(95 \% \mathrm{CI}$ $113.6-130.3 \mathrm{nmol} / \mathrm{mg} / \mathrm{min}$ ) was even higher than that in humans (95\% CI $17.40-27.07 \mathrm{nmol} / \mathrm{mg} / \mathrm{min}$ ). Age, sex, and race did not appear to affect UGT activity, nor did we observe any effects of BMI, alcohol intake, smoking habit, or drug use on UGT activity. UGT activity in the human livers was positively correlated with lipase activity $\left(r_{\mathrm{s}}=0.33\right)$.
ADH activity

Alcohol dehydrogenase activity for 2-EH was significantly higher in our human subjects than in mice, a result that differs considerably from those for lipase or UGT activity (Table 2). Mean ADH activity was $8.6 \mathrm{nmol} / \mathrm{mg} / \mathrm{min}$ in human $(95 \%$ CI 7.4-9.8) nmol/mg/min and $4.2(95 \%$ CI $3.7-4.7) \mathrm{nmol} / \mathrm{mg} / \mathrm{min}$ in mice. The values in mice were within the large range of inter-individual differences observed in humans [maximum human value $(15.5 \mathrm{nmol} /$ $\mathrm{mg} / \mathrm{min}$ ) was tenfold greater than the lowest human value $(1.5 \mathrm{nmol} / \mathrm{mg} / \mathrm{min})]$. Mean ADH activity in two alcoholics (3.0 and $1.6 \mathrm{nmol} / \mathrm{mg} / \mathrm{min}$, respectively) was significantly lower than that in the other subjects, although there were no significant differences in ADH activity between nondrinkers and drinkers (data not shown). ADH activity did not appear to be influenced by sex, race, BMI, smoking habit, or drug use, although it did tend to increase with increasing age. In non-Hispanic white subjects aged $>60$ years, ADH activity was significantly higher than that in the other age groups, except for one person who had the lowest ADH activity $(1.5 \mathrm{nmol} / \mathrm{mg} / \mathrm{min})$, with $\mathrm{ADH}$ activity in those aged $>60$ years being $>11.9 \mathrm{nmol} / \mathrm{mg} /$ min. ADH activity in humans was positively correlated with lipase $\left(r_{\mathrm{s}}=0.36\right)$ and UGT activities $\left(r_{\mathrm{s}}=0.40\right)$.

\section{ALDH activity}

Aldehyde dehydrogenase activity for 2-EH was significantly lower in our human subjects than in mice (Table 2). The values in most subjects were comparable or lower than those in mice; however, the activity of the youngest man deviated from that in mice. Mean ALDH activities were 3.6 in human (95\% CI 3.0-4.3) and 5.6 (95\% CI 4.6-6.6) in mice, and there was a 26 -fold margin between the minimum $(0.4 \mathrm{nmol} / \mathrm{mg} / \mathrm{min})$ and the maximum $(10.5 \mathrm{nmol} /$ $\mathrm{mg} / \mathrm{min}$ ). The values were significantly higher in nonHispanic white women than in non-Hispanic men in their forties. Age, race, BMI, alcohol intake, smoking habit, or drug use did not appear to affect ALDH activity.

\section{Urinary metabolites of DEHP}

The urinary metabolites of DEHP in intact mice used in this study were measured and compared with those in humans reported elsewhere [1] (Table 4). When total metabolites between the human population and mice were compared, the amount was 37 -fold higher in mice $(8.8 \mu \mathrm{mol} / \mathrm{l})$ than in humans $(0.24 \mu \mathrm{mol} / \mathrm{l})$. The average MEHP concentration in urine was much higher (geometric mean 20 -fold, median 77 -fold) in the mice in our study 
Table 4 Urinary levels of di(2-ethylhexyl)phthalate metabolites in mice and a general population of humans, and total amount of excretion of these metabolites

\begin{tabular}{|c|c|c|c|c|}
\hline \multirow[t]{2}{*}{ Urinary metabolites $^{\mathrm{a}}$} & \multicolumn{2}{|c|}{ Human } & \multicolumn{2}{|l|}{ Mouse } \\
\hline & Mean & Median & Mean & Median \\
\hline MEHP & 2.3 & 2.1 & 132.2 & 162 \\
\hline Phthalic acid and 2cx-MMHP & N.I. & N.I. & 2557.3 & 2502 \\
\hline 5oxo-MEHP & 14.4 & 15.9 & 8.3 & 2.5 \\
\hline 5cx-MEPP & 34.5 & 35.3 & $<5$ & $<5$ \\
\hline 5ОH-MEHP & 21.8 & 23.1 & N.I. & N.I. \\
\hline Sum $(\mu \mathrm{mol} / 1 \text { urine })^{\mathrm{b}}$ & 0.24 & & 8.8 & \\
\hline
\end{tabular}

Data on human urine were obtained from Colacino et al. [24] using samples of the 2003-2004 U.S. National Health and Nutrition Examination Survey [1]

The values of mice were determined by duplicate analyses

MEHP mono(2-ethylhexyl)phthalate, 2cx-MMHP mono(2-carboxymethylhexyl)phthalate, 5oxo-MEHP mono(2-ethyl-5-oxo-hexyl) phthalate, 5cx-MEPP mono(2-ethyl-5-carboxypentyl)phthalate, $5 \mathrm{OH}$ MEHP mono(2-ethyl-5-hydroxyhexyl)phthalate

${ }^{\text {a }}$ Data on urinary levels of metabolites are presented as the geometric mean and median in humans and mice (units: $\mathrm{ng} / \mathrm{ml}$ urine)

b Total amount of excretion (Sum) of these di(2-ethylhexyl)phthalate (DEHP) metabolites

than that in the U.S. general population aged $\geq 6$ years [1]. On the other hand, mono(2-ethyl-5-oxo-hexyl)phthalate (5oxo-MEHP) and 5cx-MEPP levels in urine were higher in humans than in mice (geometric mean twofold and $>18$ fold; median six and sevenfold, respectively).

\section{Discussion}

In this study, we found clear-cut individual differences (10-26 fold) in the activities of four enzymes involved in DEHP metabolism in the livers of the human subjects. These differences were not related with the time required for the transport of each sample prior to freezing. Species differences between mice and humans were also observed for all enzyme activities; however, these differences were generally smaller (two- to sevenfold) than the individual differences. Of note is that the metabolic pathway of DEHP in humans is different from that of mice, especially the MEHP oxidation pathways.

Of the four enzymes investigated, the inter-subject variability in ALDH activity was the greatest (26-fold), followed by that in UGT activity (17-fold). There are 11 isozymes (ALDH1 9, 16, 18) in the human liver [12]. Isoform(s) which may be involved in DEHP metabolism have not been investigated. However, the frequency of ALDH2 or ALDH1A genetic polymorphisms has been reported to be low in Caucasians [13-15], suggesting that the wide range in ALDH activity observed in our study was not due to polymorphisms even if these isozymes are involved in DEHP metabolism. Other ALDH isoenzymes, for which polymorphisms have not been reported, may contribute to this inter-subject variability.

A number of UGT isoforms have also been reported; of these, UGT2B7 is a major form, accounting for $84 \%$ of the total activity to produce MEHP-glucuronide-conjugated metabolites [16]. The UGT2B7 802C $>$ T polymorphism is commonly seen in North Americans [17] and is associated with UGT activity [18-20]. Therefore, the 17-fold variation in UGT activity observed in our study may be due to such polymorphisms. However, this possibility needs to be confirmed in the future. Inter-subject variability in lipase and ADH activities were tenfold each and smaller than that in ALDH and UGT activities. Thus, the former two enzymes may be very important for the evaluation of the inter-individual difference in urinary DEHP metabolites.

Of the four enzymes we investigated, there were large species differences in microsomal lipase activity (hydrolysis from DEHP to MEHP and 2-EH) and UGT activity of about 6.7- and 5.5-fold between mice and humans, respectively. We previously reported an almost 240-fold difference in hepatic microsomal lipase activity between mice and marmosets [5]. However, in the present study, microsomal lipase activity in CD-1 mice and SpragueDawley rats was similar to that observed for $\mathrm{Sv} / 129$ mice-no difference was seen in the lipase activity between mouse strains or mice and rats. These results demonstrate that the lipase activity of humans may not be similar to that of marmosets, but rather to that of mice or rats. The difference between marmosets and humans might be attributable to the kinetic parameter, $V_{\max } / K_{\mathrm{m}}$, i.e., to the intrinsic clearance of DEHP, which was much lower in marmosets in our previous study [5] than in humans in our present study. Based on these results, we suggest that lipase might play a major role in the inter-species differences between rodents and humans in DEHP metabolism and that species difference might be large. However, this may not be true given that the species differences in enzyme activities were not as great as those found in mice and marmosets. In fact, species differences in ADH and ALDH activities were only within twofold.

To determine how these species differences in enzyme activities influence the urinary metabolites of DEHP, we also compared the urinary metabolite concentrations of the intact mice used in our study with those of humans reported elsewhere [1]. As expected based on our findings of higher lipase and UGT activities in mice than in our human subjects, the average MEHP concentration in urine was much higher in mouse urine in the present study than in the U.S. general population aged $\geq 6$ years [1]. When metabolites of DEHP in mice were assumed to be only MEHP, 
2cx-MMHP, 5oxo-MEHP, and 5cx-MEPP, the total concentration excreted in urine was $8.8 \mathrm{nmol} / \mathrm{l}$, which was 37 -fold higher in mice than in humans $(0.24 \mathrm{nmol} / \mathrm{ml})$. In another survey [21], eight metabolites of DEHP (MEHP, oxidized or carboxylated metabolites) in urine from 129 U.S. citizens were measured. The concentration of the eight metabolites in total was $0.17 \mathrm{nmol} / \mathrm{ml}$, suggesting that the species difference in DEHP metabolite levels between mice and humans was much greater than that observed for the activity of each enzyme separately in vitro. The intake of DEHP in these generations was estimated to be $8.2-18.9 \mu \mathrm{g} / \mathrm{kg}$ body weight (b.w.)/day [6]. In contrast, the DEHP intake of the mice used in the present study was estimated to be $11.6-51.1 \mu \mathrm{g} / \mathrm{kg}$ b.w./day because the concentrations of DEHP were 116-511 ng/g in rodent feed and were not detected in drinking water [22]. Thus, DEHP exposure levels between mice and humans can be considered to be comparable, and the difference in urinary MEHP levels between them might not have resulted from DEHP exposure levels.

On the other hand, 5oxo-MEHP and 5cx-MEPP levels in the urine were higher in humans than mice. In these metabolic pathways, not only CYP but also ADH and ALDH are involved [23]. Since the species difference in the latter two enzymes was not as great as for those mentioned above, the difference in CYP activities for DEHP metabolism may be great. In particular, the concentrations of 5 cx-MEPP were quite different between humans and mice. This finding is supported by a previous report that human CYP2C $9 * 1$ and CYP2C $9 * 2$ mainly produce this form [7]. CYP4A is also involved in the formation of mono(2-ethyl5-hydroxyhexyl)phthalate (5OH-MEHP); however, its activity is very weak [7]. Therefore, the contribution of this enzyme can be ignored. In contrast, the level of $2 \mathrm{cx}-$ MMHP in human urine has been found to be $5.2 \mathrm{ng} / \mathrm{ml}$ [21], although this metabolite was not measured by Colacino et al. [24]. This value is significantly lower than that found in mice and lower than the values for 5cx-MEPP and 5OH-MEHP, suggesting that 2cx-MMHP is not a major metabolite in humans.

Taken together, we standardly observed species differences in urinary DEHP metabolites such as MEHP and in its oxidative metabolites between mice and humans: (1) MEHP to 2cx-MMHP was a major metabolic pathway in mice, whereas MEHP to 5oxo-MEHP and 5cx-MEPP was a major pathway in humans; (2) MEHP to 2cx-MMHP was only a minor pathway in humans (Fig. 2). Species differences in the activities of lipase and UGT may have contributed to the urinary MEHP level, while that of CYP may have contributed to MEHP oxidative metabolites.

In risk assessments of chemical substances, tenfold has usually been used as an uncertainty factor when considering the risk level from animal experiments to human, but in

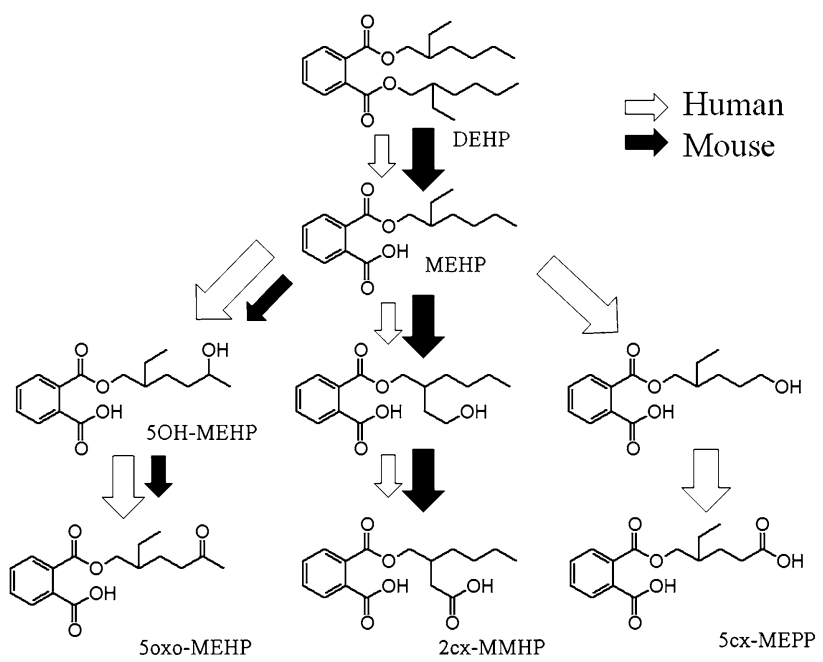

Fig. 2 Major di(2-ethylhexyl)phthalate (DEHP) metabolic pathways and metabolites referred to in this study. White and black arrows Possible pathway DEHP metabolic pathways in mouse and humans, respectively, size of arrow Metabolic capacity of each step. See Abbreviations for terms

some cases, a value of 2.5 -fold has been used when the kinetics between species were similar [25]. As mentioned above, the metabolic pathway of DEHP in addition to the metabolic activity, i.e. kinetics of DEHP, was quite different between mice and humans, therefore, a 10-fold rather than 2.5 -fold value, or much higher values, were adequate as an uncertain factor of species difference. On the other hand, a 10-fold value was also commonly used as an uncertainty factor of inter-individual difference in the kinetics and dynamics of chemical substances. It may be very difficult to estimate the difference from the results of urinary metabolites in humans because there was no information regarding exposure levels. Considering from our results, inter-individual differences in enzyme activities were much greater than species differences. Therefore, at least a 10 -fold human variability may be required in the risk assessment of DEHP.

In the present study, ADH activity for 2-EH tended to be higher in the elderly. However, no effects of age have been reported on ADH activity for ethanol in human liver [26]. Isoenzyme ADH IV activity, but not isoenzyme ADH I and III activities, has been reported to decrease with aging [27]. Our data suggest that the ADH activities for both 2-EH and ethanol increased with aging. The reason for this discrepancy between current and former studies is unclear, and studies involving large numbers of white male subjects will be needed to confirm if ADH activity actually does increase with aging in the white male population.

There are some limitations to our study. First, we could not obtain a sufficient number of liver samples from Hispanics, African Americans, and Asians. In addition, accurate data on the consumption of cigarettes, alcohol, and 
drugs were not available. Therefore, the effects of these substances on the enzyme activities we investigated could not be accurately evaluated. Further study using additional samples is needed to draw conclusions on the sexual and possible aging effect on ALDH and ADH activities, respectively, as well as on racial differences and the effects of habitual use of substances on DEHP metabolism. Additionally, in our study we only used 2-EH and 2-ethylhexanal were used as substrates for ADH or ALDH, respectively. Notably, ADH and ALDH also play a role in oxidative pathways of MEHP. It is doubtful whether the activities for 2-EH and 2-ethylhexanal apply to those in the steps of MEHP-derived oxidation.

In conclusion, species differences in DEHP metabolism between humans and mice were clearly observed, but they were much smaller than those between marmosets and mice [5]. We found that the human liver had a lower DEHP-metabolizing capacity than the mouse, except for those steps mediated by ADH, which may be responsible for the urinary metabolites. Inter-individual differences in DEHP metabolism in humans may be more crucial than the species difference. Notably, our data are useful in the context of the risk of DEHP-induced toxicity.

Acknowledgments This study was supported by a research grant from the Food Safety Commission, Japan (1002). We thank Dr. Satoshi Suzuki (HAB Research Organization) for facilitating the procedure to obtain human livers from National Disease Research Interchange (NDRI), USA.

Conflict of interest None.

\section{References}

1. Center for Disease Control and Prevention (CDC). The Fourth National Report on Human Exposure to Environmental Chemicals, Updated Tables. 2012. CDC, Atlanta.

2. Albro PW, Lavenhar SR. Metabolism of di(2-ethylhexyl)phthalate. Drug Metab Rev. 1989;21(1):13-34.

3. Koch HM, Preuss R, Angerer J. Di(2-ethylhexyl)phthalate (DEHP): human metabolism and internal exposure-an update and latest results. Int J Androl. 2006;29(1):155-65 (discussion 181-5).

4. Ito Y, Yamanoshita O, Kurata Y, Kamijima M, Aoyama T, Nakajima T. Induction of peroxisome proliferator-activated receptor alpha (PPARalpha)-related enzymes by di(2-ethylhexyl)phthalate (DEHP) treatment in mice and rats, but not marmosets. Arch Toxicol. 2007;81(3):219-26.

5. Ito Y, Yokota H, Wang R, Yamanoshita O, Ichihara G, Wang H, et al. Species differences in the metabolism of di(2-ethylhexyl)phthalate (DEHP) in several organs of mice, rats, and marmosets. Arch Toxicol. 2005;79(3):147-54.

6. Kavlock R, Barr D, Boekelheide K, Breslin W, Breysse P, Chapin $\mathrm{R}$, et al. NTP-CERHR expert panel update on the reproductive and developmental toxicity of di(2-ethylhexyl)phthalate. Reprod Toxicol. 2006;22(3):291-399.

7. Choi K, Joo H, Campbell JL Jr, Clewell RA, Andersen ME, Clewell HJ 3rd. In vitro metabolism of di(2-ethylhexyl)phthalate
(DEHP) by various tissues and cytochrome P450s of human and rat. Toxicol In Vitro. 2012;26(2):315-22.

8. Wang RS, Nakajima T, Honma T. Trichloroethylene inhibits aldehyde dehydrogenase only for aliphatic aldehydes of short chains in rats. Toxicology. 1999;132(1):9-18.

9. Hayashi Y, Ito Y, Yanagiba Y, Kamijima M, Naito H, Nakajima T. Differences in metabolite burden of di(2-ethylhexyl)phthalate in pregnant and postpartum dams and their offspring in relation to drug-metabolizing enzymes in mice. Arch Toxicol. 2012;86(4): 563-9.

10. Kawai T, Mizunuma K, Yasugi T, Uchida Y, Ikeda M. The method of choice for the determination of 2,5-hexanedione as an indicator of occupational exposure to $n$-hexane. Int Arch Occup Environ Health. 1990;62(5):403-8.

11. Finkelstein MM, Verma DK. Exposure estimation in the presence of nondetectable values: another look. AIHAJ. 2001;62(2):195-8.

12. Marchitti SA, Brocker C, Stagos D, Vasiliou V. Non-P450 aldehyde oxidizing enzymes: the aldehyde dehydrogenase superfamily. Expert Opin Drug Metab Toxicol. 2008;4(6): 697-720.

13. Goedde HW, Agarwal DP, Harada S, Rothhammer F, Whittaker JO, Lisker R. Aldehyde dehydrogenase polymorphism in North American, South American, and Mexican Indian populations. Am J Hum Genet. 1986;38(3):395-9.

14. Goedde HW, Agarwal DP, Harada S, Meier-Tackmann D, Ruofu $\mathrm{D}$, Bienzle $\mathrm{U}$, et al. Population genetic studies on aldehyde dehydrogenase isozyme deficiency and alcohol sensitivity. Am J Hum Genet. 1983;35(4):769-72.

15. Spence JP, Liang T, Eriksson CJ, Taylor RE, Wall TL, Ehlers $\mathrm{CL}$, et al. Evaluation of aldehyde dehydrogenase 1 promoter polymorphisms identified in human populations. Alcohol Clin Exp Res. 2003;27(9):1389-94.

16. Joo H, Choi K, Grimes J, O'Connell T, Clewell R, Campbell J, Clewell H. Phase II biotransformation of di(2-ethylhexyl)phthalate in human and rat. In: 50th Annu Meet Society of Toxicology. 2011. Article no. 1824.

17. Mehlotra RK, Bockarie MJ, Zimmerman PA. Prevalence of UGT1A9 and UGT2B7 nonsynonymous single nucleotide polymorphisms in West African, Papua New Guinean, and North American populations. Eur J Clin Pharmacol. 2007;63(1): $1-8$.

18. Innocenti F, Liu W, Fackenthal D, Ramirez J, Chen P, Ye X, et al. Single nucleotide polymorphism discovery and functional assessment of variation in the UDP-glucuronosyltransferase 2B7 gene. Pharmacogenetics Genomics. 2008;18(8):683-97.

19. Girard H, Court MH, Bernard O, Fortier LC, Villeneuve L, Hao $\mathrm{Q}$, et al. Identification of common polymorphisms in the promoter of the UGT1A9 gene: evidence that UGT1A9 protein and activity levels are strongly genetically controlled in the liver. Pharmacogenetics. 2004;14(8):501-15.

20. Olson KC, Dellinger RW, Zhong Q, Sun D, Amin S, Spratt TE, et al. Functional characterization of low-prevalence missense polymorphisms in the UDP-glucuronosyltransferase 1A9 gene. Drug Metab Dispos. 2009;37(10):1999-2007.

21. Silva MJ, Reidy JA, Preau JL, Samandar E, Needham LL, Calafat AM. Measurement of eight urinary metabolites of di(2-ethylhexyl)phthalate as biomarkers for human exposure assessment. Biomarkers. 2006;11(1):1-13.

22. Japan Ministry of Health, Labour and Welfare. Advisory Committee on Health Effects of Endocrine Disruptors. 2005.

23. Albro PW. Absorption, metabolism, and excretion of di(2-ethylhexyl)phthalate by rats and mice. Environ Health Perspect. 1986;65:293-8.

24. Colacino JA, Harris TR, Schecter A. Dietary intake is associated with phthalate body burden in a nationally representative sample. Environ Health Perspect. 2010;118(7):998-1003. 
25. Dourson ML, Felter SP, Robinson D. Evolution of science-based uncertainty factors in noncancer risk assessment. Regul Toxicol Pharmacol. 1996;24(2 Pt 1):108-20.

26. Wynne HA, Wood P, Herd B, Wright P, Rawlins MD, James OF. The association of age with the activity of alcohol dehydrogenase in human liver. Age Ageing. 1992;21(6):417-20.
27. Matsumoto M, Yokoyama H, Shiraishi H, Suzuki H, Kato S, Miura S, et al. Alcohol dehydrogenase activities in the human gastric mucosa: effects of Helicobacter pylori infection, sex, age, and the part of the stomach. Alcohol Clin Exp Res. 2001;25[6 Suppl]:29S-34S. 